\title{
Anterior cruciate ligament tears in pediatric patients: Current concepts
}

\author{
Gregorio A. Villarreal-Villarreal*, Carlos A. Acosta-Olivo, Félix Vilchez-Cavazos, \\ Santiago De la Garza-Castro, José F. de la Garza-Salazar and Victor M. Peña-Martinez \\ Department of Orthopedics and Traumatology, "Dr. José E. Gonzalez" University Hospital, Autonomous University of Nuevo Leon, Monterrey, \\ Nuevo Leon, Mexico
}

\begin{abstract}
Anterior cruciate ligament (ACL) tears on pediatrics patients are clearly on the rise. This rise is multifactorial and can be attributed to an increase in sports participation, sports specialization, year-long training, and competitions. The diagnosis of this entity is clinical and confirmed with a magnetic resonance imaging. Historically, ACL ruptures in pediatric patients have been treated non-surgically due to a fear of growth disturbances. This leads to poor results in the long-and medium-term follow-up. Surgical treatment has gained popularity with relatively good outcomes; several reconstruction techniques have been described, with or without damaging the physis. In this review article, we present the most recent epidemiological data and treatment options.
\end{abstract}

Key words: Anterior cruciate ligament tears. Pediatrics. Physeal sparing techniques. Transphyseal techniques.

\section{Introduction}

Intrasubstance anterior cruciate ligament (ACL) tears in pediatric patients were considered rare injuries ${ }^{1}$, along with fractures and avulsions of the tibial spine as equivalent entities ${ }^{2}$. A rise in the amount of high-performance young athletes has resulted in a greater number of ACL injuries in patients with active stages of growth (open physes). The treatment of these patients has always been a controversial topic; there are those who prefer conservative therapy and those who opt for surgical management ${ }^{2}$. Nevertheless, to this day, there are no treatment guidelines or specific instructions to treat the problem ${ }^{1-3}$
This review article covers aspects of the anatomy, physical examination, and diagnosis of ACL ruptures in pediatric patients, as well as the different treatment options.

\section{Epidemiology}

To this day, there is no real incidence of $A C L$ injuries in pediatric patients. However, when we are faced with a traumatic knee injury with data of acute hemarthrosis, it is estimated that between 10 and $65 \%$ have an $\mathrm{ACL}$ rupture/injury 4 . An epidemiological study of athletes under 20 years old in the state of New York who were treated through an $\mathrm{ACL}$ reconstruction revealed an

\section{Correspondence:}

*Gregorio A. Villarreal-Villarreal

E-mail: villarrealga@gmail.com
Available online: 30-09-2019

Date of reception: 18-03-2019

Date of acceptance: 28-05-2019 DOI: 10.24875/RMU. 19000082
Medicina Universitaria. 2019;21(3):125-130 www.medicinauniversitaria.org CC BY-NC-ND license (http://creativecommons.org/licenses/by-nc-nd/4.0/). 
increase from $17.6 \%$ in 1990 to $50.9 \%$ in 2009 , with a higher incidence in 17-year-old patients. These data do not show a real incidence of the injury; however, indirectly through $\mathrm{ACL}$ reconstructions, it is considered to show an increase in ACL injuries ${ }^{5}$. This could be a consequence of the increase in sports activity among young people, training/competitions, as well as sports specialization at an early age. Moreover, awareness and knowledge of early diagnosis on behalf of trainers, physical therapists, and family members is worth noting ${ }^{6,7}$.

\section{Risk factors for ACL ruptures}

To identify the group of skeletal immature patients with ACL ruptures, it is important to identify the intrinsic and extrinsic factors. Among the intrinsic factors, there are mechanical, hormonal, and anatomical ${ }^{1}$. Anatomical risk factors are the most studied; these include the increase in pelvic tilt, increase in femoral anteversion, increase of the $Q$ angle, increase in the fall of the tibial slope, increase in the pronation of the foot and scaphoid fall, and a decrease in the width of the femoral intercondylar notch ${ }^{1,8}$. Another risk factor is the female gender, where an ACL rupture is 6 times more frequent compared to males ${ }^{8}$. Female patients are usually accompanied by data of hypermobility-hyperlaxity, genu valgus, and genu recurvatum ${ }^{9}$. In addition, ACL ruptures are linked to phases of the menstrual cycle, specifically the pre-ovulation or follicular phase, where estrogen concentration plays a major role ${ }^{8}$.

Among the extrinsic factors, the most important are weather conditions, footwear, and interaction of the footwear with the field/track. Regarding the field/track, light rain and evaporation during summer make them harder, increasing sprains in the ACL. Concerning footwear, size, height, and position of the spikes (specifically in the lateral margin in the sole of the spikes) increase ACL sprains ${ }^{1,8}$.

\section{Physical examination and diagnosis of ACL rupture in pediatric knees}

\section{Physical examination}

The pediatric patient usually refers to a forced varum valgum with a twist, referring to an audible "pop" or a sensation of "popping" in the affected knee. Physical examination ought to be complete and directed toward the injury, inspection, palpation, and clinical maneuvers specific to an ACL rupture. At inspection, local ecchymosis data, the patient's antalgic position, and pain facies should be taken into account. Another important point of data at the inspection is the axis alignment of the limbs; in skeletally immature patients, it is typical to find the alignment in valgum of both knees ${ }^{9}$.

At palpation, one of the most significant clinical signs is hemarthrosis. The presence of an acute hemarthrosis is suggestive of an ACL rupture in $65 \%$ of cases ${ }^{4}$.

Among the most important clinical maneuvers to detect ACL insufficiency, there are the Lachman test, which is performed with the patient in dorsal decubitus on the examination table, with the knee flexed $30^{\circ}$ in external rotation, with a hand holding the distal end of the thigh on the external side, and, on the other hand, the upper end and inner side of the proximal tibia. With the thumb of the tibial hand placed over the tibial tuberosity, they should both move simultaneously, the tibia forward and the thigh backward, observing the degree of anterior translation of the tibia. The anterior drawer test is performed, placing the patient in the supine position with the knees bent at a $90^{\circ}$ angle, and the feet flat on the examination table. Hands are placed around the knee with the thumbs over the medial and lateral articular line, and the index fingers toward the front, if positive, a displacement under the femur is observed.

The pivot-shift test is conducted with the patient again in the supine position, with the hips flexed at a $30^{\circ}$ angle (passive form) and an abduction of $30-40^{\circ}$ of the hip, to relax the iliotibial band (IB) and allow the tibia to rotate. The examiner places himself/herself at the lateral side of the involved knee, the leg, and ankle are grasped with one hand, keeping the internal rotation of the tibia at a $20^{\circ}$ angle. The opposite hand is placed on the lateral side of the leg at a proximal tibioperoneal articulation level to increase valgus stress and internal rotation. Maintaining internal rotation, valgus stress is applied to the knee while the knee is being slowly flexed. If there is an anterior subluxation of the tibia, the test will be positive". Recently, the "Lever Sign/Lelli test" was described with $100 \%$ sensitivity in complete, partial, acute, and chronic tears of the ACL. This test is conducted with the patient supine, with the knees extended on the table. The examiner stands on the side of the knee to examine, placing the closed fist under the proximal third of the calf, causing the knee to flex slightly $\left(20-30^{\circ}\right)$. The other hand is placed over the distal third of the quadriceps muscle, applying downward force to the quadriceps. With this configuration, the patient's leg acts as a lever over a fulcrum in the fist of the examiner. In an intact knee, the ACL acts 
as a lever due to the downward force applied over the quadriceps; the knee goes toward full extension, and the heel is elevated from the examination table. In a knee with a ruptured ACL, there is no lever between the applied force to the quadriceps and the fist under the calf; hence, the heel is not elevated from the table ${ }^{10}$. However, this clinical sign has not yet been evaluated in pediatric patients. It is worth mentioning that these maneuvers during the acute period of the injury are difficult to explore due to the pain and inflammation. Other important clinical data are signs of hypermobility, as well as the genu recurvatum, which is very common in pediatric patients ${ }^{9}$.

\section{Imaging studies}

The radiographic study of skeletally immature patients begins with simple X-rays (anteroposterior and lateral), complemented with telemetry of the lower limbs in a single projection, which includes everything from the hips to the ankles, allowing us to assess three things: (1) discrepancy in the length of extremities, (2) angular deformities, and (3) the patient's mechanical axis in coronal angle ${ }^{1}$. However, simple $\mathrm{X}$-rays give us little information about ACL insufficiency. The best imaging study to evaluate an $A C L$ rupture is magnetic resonance imaging (MRI), which has a proven sensitivity of $95 \%$ and a specificity of $88 \%$. The most important primary data are an abnormal course of the ligament, ligament discontinuity, and abnormalities in the intensity of the images like a diffuse increase in the $\mathrm{ACL}$ signal in $\mathrm{T} 2^{11}$.

Another major point is the evaluation of bone age or skeletal maturity. For this, it is necessary to obtain anteroposterior X-rays of the left hand, and using the Greulich and Pyle Atlas as a reference, will allow us to estimate the skeletal age of the patient ${ }^{1}$.

\section{Treatment}

\section{Non-surgical}

Treatment for these injuries historically has been non-surgical and to wait for complete physeal closure. This treatment is based on the placement of a knee brace, physical therapy, and modifications in physical activities $^{12}$. The main justification for conservative treatment is avoiding damaging the growth physes during surgery and not generating future length discrepancies in the limbs or residual knee malformations (valgus and recurvatum) ${ }^{3}$. These malformations are a result of the transgression of the growth physes and the perichondral ring with drill bits, staples, interferential screws, and even by bone plugs used in the same ACL graft ${ }^{13,3}$.

Another reason to justify a conservative treatment is the regenerative ability which pediatric patients possess; nevertheless, in ACL injuries, this has not been a positive thing. In a study by Kocher et al., they analyzed conservative treatment in 45 patients with partial $A C L$ injuries; the treatment consisted of the use of a knee brace, minimum stress on the leg for a period of 6-8 weeks, a specific rehabilitation program, and a return to sports in 3 months. They found that up to a third of the patients $(31 \%)$ required surgical intervention due to a sensation of instability and failure to practice sports ${ }^{14}$.

In general, conservative treatment for pediatric patients with ACL ruptures has led to poor results. Henry et al. compared the results of two groups; Group 1, early surgery and Group 2, late surgery, which included a total of 56 patients and proved that the patients in Group 2 (late surgery) showed a greater risk of meniscus rupture (41\%) versus Group $1(16 \%)$, as well as a greater rate of meniscectomies versus meniscal repairs $^{15}$. Lawrence et al. set a time limit for ACL reconstruction in skeletally immature individuals of 12 weeks, finding that after this period, there was a considerable increase in irreparable injuries of the medial meniscus and chondral injuries in lateral and patellofemoral compartment ${ }^{6}$.

There are recommendations for non-surgical treatment in patients with ACL injuries, and they are patients under 14 years of age with normal Lachman and pivot-shift tests ${ }^{14}$. Taking into consideration, the previous results and reviewed literature to this point, it is very hard to obtain these three criteria and recommend conservative treatment.

\section{Surgical}

Primary surgical treatment has become a common practice among orthopedics, given the poor results of conservative treatment, as mentioned above. When we talk about surgical treatments, it is important to assess these patients with the Tanner scale of maturation of secondary sexual characteristics (male and female). This classification is subdivided into four Stages I-IV ${ }^{16}$.

There are four surgical techniques for ACL reconstruction in pediatric patients: (1) physeal sparing: IT band; (2) transphyseal; (3) partially epiphyseal; and (4) full epiphyseal ${ }^{17}$ (Fig. 1: different techniques of pediatric $A C L$ reconstruction). 


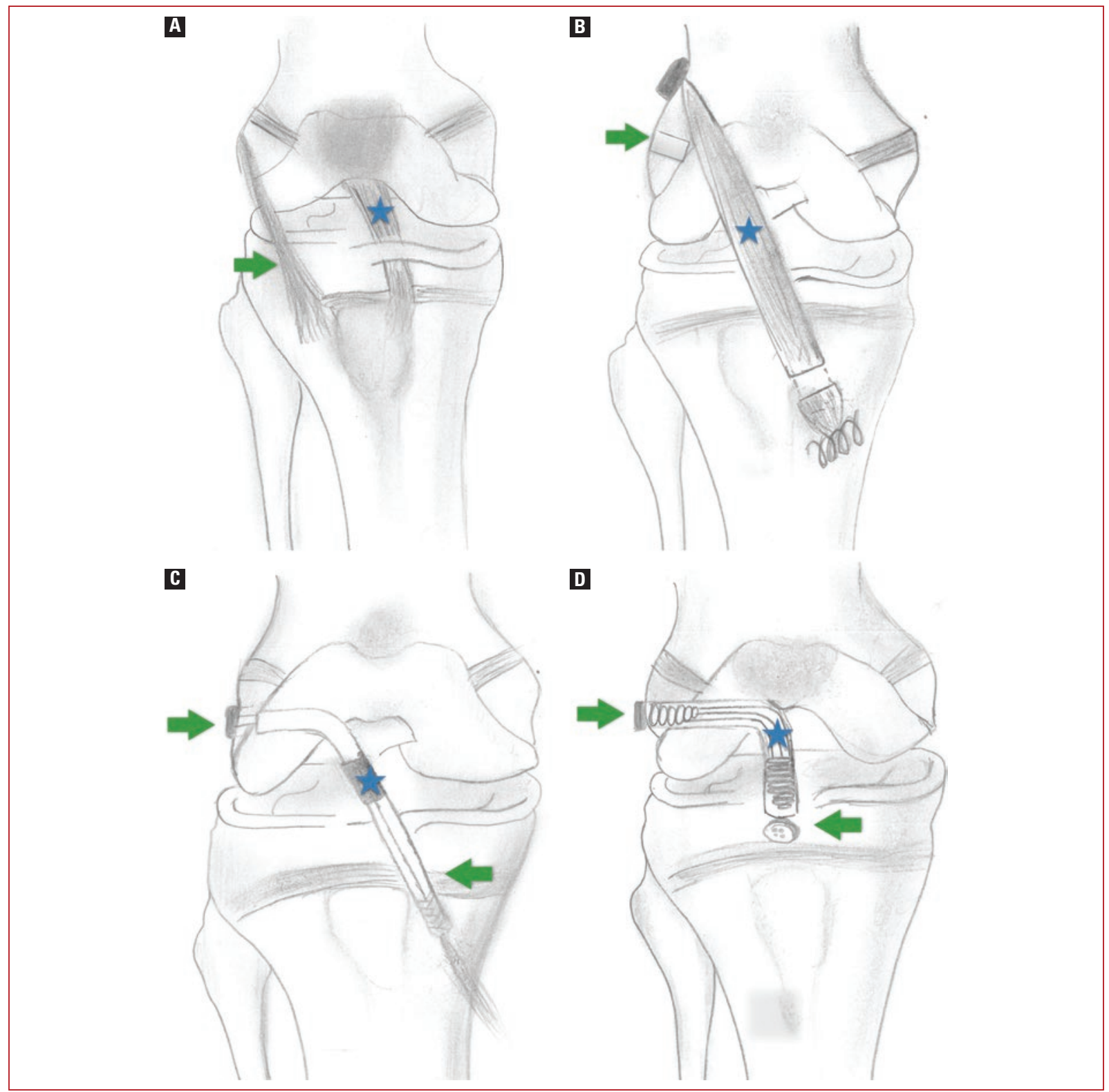

Figure 1. Different reconstruction techniques of the pediatric anterior cruciate ligament. A: extraphyseal technique: iliotibial band (blue star indicates the anterior cruciate ligament, a green arrow indicates the iliotibial band);

B: transphyseal (the blue star marks the new anterior cruciate ligament, the green arrow indicates the physis of growth, which was traversed.); C: partial epiphyseal (the blue star points to the new anterior cruciate ligament, the femoral/superior green arrow points to the fixation below the growth physis, the lower green arrow points to the lower physis, traversed by the new anterior cruciate ligament); D: full epiphyseal (the blue star points to the new anterior cruciate ligament, the upper green arrow points to the fixation system below the growth physis, and the lower green arrow indicates the fixation system above the physis of growth).

The surgical technique, which has overcome the test of time with positive results, is the one by Kocher et al. This is a physeal-sparing technique (without damage to the growth physes), which is the technique of choice in patients with a Tanner I or II. In this technique, through a wound on the lateral face of the distal calf to the IB; the same (IB) band (1 $\mathrm{cm}$ wide and $10 \mathrm{~cm}$ long) is placed, leaving the distal end inserted in the Gerdy's tubercle. That same band goes behind the intercondylar zone in the over-the-top position, then through the intermeniscal ligament, and is finally sutured to the periosteum of the proximal epiphysis of the tibia. 
The first report of this technique dates from 2005. Forty-four patients were studied from 1980 to 2002. There were two graft failures, but positive results on clinical scales such as IKDC, which reported $96.7( \pm 6)$ points, while the Lysholm score reported $95.7( \pm 6.7)$ points, without reporting abnormalities in growth².

Recently, the same author, increasing the number to 237 patients, obtained the following results: $96.8 \%$ Grade $A$ in the Lachman A test (IKDC-Lachman A> 1-2 mm; B 3-5 mm, C 6-10 mm; D >10 mm), 98.8\% Grade $A$ in the pivot shift (IKDC-pivot shift A normal; B sliding; $C$ clunk; $D$ severe), with $6.6 \%$ graft failure, $\mathrm{Pe}$ di-IKDC $93.3( \pm 11)$, and Lysholm $93.4( \pm 9.9)$. However, $48 \%$ of the patients reported an asymmetry in the thigh though only $1.6 \%$ reported pain at the site of the graft ${ }^{7}$.

Transphyseal reconstruction is used for patients of Tanner I through IV. This technique is based in drilling through the tibial and femoral physes, normally using the ACL transtibial reconstruction technique ${ }^{18}$. The graft is of the surgeon's choice; it may be bone-patellar tendon-bone (BPTB) or hamstrings. From these, BPTB is the one which is more associated with physeal arrests due to bone plugs. In addition, bone plugs of the proximal tibia can cause recurvatum in the tibia'. Recently, physeal arrest problems were calculated in a meta-analysis, finding a rate of $1.9 \%$ with the transphyseal technique. From the used grafts, the higher rate of failure is seen with the hamstring graft compared to BPTB. However, the most observed complication with the latter is physeal arrest ${ }^{19}$. The rerupture and plasty failure rate have been observed at $4.2 \%$, independently of the used graft ${ }^{19}$. Clinical results at medium and longterm are good, obtaining an IKDC of 91.5 points and a Lysholm score of 93.5, in addition to a return to previous sports activities of $88.8 \%{ }^{1}$.

A revision by Palleta et al. described the standards for a successful surgery using the transphyseal technique, which are as follows: (1) hamstring muscle graft, (2) low-speed drill bit and high torque, and (3) central, vertical, and good length tunnels ${ }^{18}$.

The full epiphyseal technique has been used in patients with Tanner Stages I-III. This technique is based on piercing the tunnels of the new ACL through the tibial and femoral epiphyses without damaging the growth zones (physes), obtaining the position of the new plasty almost anatomically ${ }^{1}$. The preferred graft is the hamstring autograft, using only the semitendinosus in a quadrupled way. Today, the most utilized fixation system is with buttons on both femoral and tibial corticals or a screw in the tibial epiphyses ${ }^{20,13}$.
The most common surgical technique, with positive results up to 2 years today, is the one by Cordasco et $\mathrm{a}^{20}$. In his latest publication of 23 patients ( 6 females and 17 males) using the same technique with buttons in corticals on both epiphyses and the same autograft (semi-tendinous quadrupled), they obtained the following results: IKDC 96.4 points, Lysholm 97.9 points, and negative Lachman and pivot-shift tests in all patients. So far, two failures in ACL plasty have been reported, along with an average return to sports activity of 13.5 months ${ }^{21}$. This technique, just as the rest of the full epiphyseal techniques described above, is complemented with the help of intraoperative fluoroscopy, while some authors even describe the use of computed tomography ${ }^{13}$.

Today, there are several surgical techniques described for $\mathrm{ACL}$ reconstruction in pediatric patients, all with positive results. The preference for each technique will depend on the surgeon ${ }^{17}$. However, Kocher et al. have a list of recommendations for primary surgical management of these patients: (1) rupture over $50 \%$ of ACL thickness in the MRI, (2) rupture of the posterolateral fascicle, (3) pivot-shift ( \pm ) test Grade B or greater, and (4) a skeletal age of over 14 years.

\section{Rehabilitation and return to sports}

After ACL reconstruction surgery, regardless of the technique used, a specific rehabilitation protocol ought to be performed. Fabricant et al. recommend following three phases: Phase I: within the first 4 weeks, the objectives are a full extension and a passive flexion of $90^{\circ}$, mobility of the patella, and improvement in the contracture of the quadriceps and hamstrings. Phase II: covers from week 4 to week 8 , the objectives are a full extension and flexion of $125^{\circ}$, gait reeducation, and full neuromuscular control when bearing weight in the operated leg without pain. Phase III: from week 8 to week 16, the objectives are full mobility, strength improvement, and volume of the quadriceps and trunk. Phase IV: covers from week 16 to week 20, the objectives are to recover strength in the quadriceps and hamstrings. Phase V: covers from week 20 to week 28 , it is characterized by the recovering of the ability to run without pain, full flexibility, and dynamic control of jumping and landing on the affected knee. Moreover, exercises specific to a sport are put in place, supervised by a physical therapist. The final post-operative phase begins at week 28 and continues until the patient returns to their sport ${ }^{1}$. Finally, the return to sports should not be based in a matter of time, but on the functional abilities of the patient. For example, performing a 
horizontal jump, a "single-leg hop test" and isokinetic tests are necessary to return to sports ${ }^{21}$. To accomplish this, multidisciplinary management of the patient is essential, especially when regaining neuromuscular control of the knee in a pediatric patient. Recently, Cordasco et al. determined that the return to a sport ought to be judged by accomplishing objectives and not by time of evolution of the surgery. The average time to return to a sport was 13.5 months $^{21}$.

\section{Conclusions}

ACL rupture incidence in pediatric patients is on the rise, but to this day, there is not any concrete epidemiological data on this topic. This is a result of an increase in sports activity in these patients. Management of these patients historically has been conservative; however, results in the short, medium, and long term have not been favorable due to an increase in chondral and meniscal injuries. Today, surgical treatment for these patients is becoming more and more common; there are four different techniques: extra-articular, extraphyseal, transphyseal, and partially transphyseal; all of which show positive results. With proper rehabilitation and multidisciplinary management of the pediatric patient, a patient who has undergone ACL reconstruction can return to their sports activity at their respective level of activity previous to the injury.

\section{Conflicts of interest}

The authors declare that does not exist conflicts of interest.

\section{Ethical disclosures}

Protection of human and animal subjects. The authors declare that no experiments were performed on humans or animals for this study.

Confidentiality of data. The authors declare that they have followed the protocols of their work center on the publication of patient data.

Right to privacy and informed consent. The authors declare that no patient data appear in this article.

\section{References}

1. Fabricant PD, Jones KJ, Delos D, Cordasco FA, Marx RG, Pearle AD, et al. Reconstruction of the anterior cruciate ligament in the skeletally immature athlete: a review of current concepts: aAOS exhibit selection. J Bone Joint Surg Am. 2013;95:e28.

2. Kocher MS, Garg S, Micheli LJ. Physeal sparing reconstruction of the anterior cruciate ligament in skeletally immature prepubescent children and adolescents. J Bone Joint Surg Am. 2005;87:2371-9.

3. Kocher MS, Saxon HS, Hovis WD, Hawkins RJ. Management and complications of anterior cruciate ligament injuries in skeletally immature patients: survey of the herodicus society and the ACL study group. J Pediatr Orthop. 2002;22:452-7.

4. Eiskjaer S, Larsen ST, Schmidt MB. The significance of hemarthrosis of the knee in children. Arch Orthop Trauma Surg. 1988;107:96-8.

5. Dodwell ER, Lamont LE, Green DW, Pan TJ, Marx RG, Lyman S, et al. 20 years of pediatric anterior cruciate ligament reconstruction in New York state. Am J Sports Med. 2014;42:675-80.

6. Lawrence JT, Argawal N, Ganley TJ. Degeneration of the knee joint in skeletally immature patients with a diagnosis of an anterior cruciate ligament tear: is there harm in delay of treatment? Am J Sports Med. 2011;39:2582-7

7. Kocher MS, Heyworth BE, Fabricant PD, Tepolt FA, Micheli LJ. Outcomes of physeal-sparing $A C L$ reconstruction with iliotibial band autograft in skeletally immature prepubescent children. J Bone Joint Surg Am. 2018;100:1087-94.

8. Alentorn-Geli E, Myer GD, Silvers HJ, Samitier G, Romero D Lázaro-Haro C, et al. Prevention of non-contact anterior cruciate ligament injuries in soccer players. Part 1: mechanisms of injury and underlying risk factors. Knee Surg Sports Traumatol Arthrosc. 2009;17:705-29.

9. Hinton RY, Rivera VR, Pautz MJ, Sponseller PD. Ligamentous laxity of the knee during childhood and adolescence. J Pediatr Orthop. 2008;28:184-7

10. Lelli A, Di Turi RP, Spenciner DB, Dòmini M. The "Lever sign": a new clinical test for the diagnosis of anterior cruciate ligament rupture. Knee Surg Sports Traumatol Arthrosc. 2016;24:2794-7.

11. Lee K, Siegel MJ, Lau DM, Hildebolt CF, Matava MJ. Anterior cruciate ligament tears: $\mathrm{mR}$ imaging-based diagnosis in a pediatric population. Radiology. 1999;213:697-704.

12. Woods GW, O'Connor DP. Delayed anterior cruciate ligament reconstruction in adolescents with open physes. Am J Sports Med. 2004; 32:201-10.

13. Lawrence JT, Bowers AL, Belding J, Cody SR, Ganley TJ. All-epiphyseal anterior cruciate ligament reconstruction in skeletally immature patients. Clin Orthop Relat Res. 2010;468:1971-7.

14. Kocher MS, Micheli LJ, Zurakowski D, Luke A. Partial tears of the anterior cruciate ligament in children and adolescents. Am J Sports Med. 2002;30:697-703.

15. Henry J, Chotel F, Chouteau J, Fessy MH, Bérard J, Moyen B, et al. Rupture of the anterior cruciate ligament in children: early reconstruction with open physes or delayed reconstruction to skeletal maturity? Knee Surg Sports Traumatol Arthrosc. 2009;17:748-55.

16. Tanner JM, Whitehouse $\mathrm{RH}$. Clinical longitudinal standards for height, weight, height velocity, weight velocity, and stages of puberty. Arch Dis Child. 1976;51:170-9.

17. Milewski MD, Beck NA, Lawrence JT, Ganley TJ. Anterior cruciate ligament reconstruction in the young athlete: a treatment algorithm for the skeletally immature. Clin Sports Med. 2011;30:801-10.

18. Paletta GA Jr. Complete transphyseal reconstruction of the anterior cruciate ligament in the skeletally immature. Clin Sports Med. 2011;30:779-88.

19. Frosch $\mathrm{KH}$, Stengel D, Brodhun T, Stietencron I, Holsten D, Jung C, et al. Outcomes and risks of operative treatment of rupture of the anterior cruciate ligament in children and adolescents. Arthroscopy. 2010; 26:1539-50.

20. McCarthy MM, Graziano J, Green DW, Cordasco FA. All-epiphyseal, all-inside anterior cruciate ligament reconstruction technique for skeletaIly immature patients. Arthrosc Tech. 2012;1:e231-9.

21. Cordasco FA, Mayer SW, Green DW. All-inside, all-epiphyseal anterior cruciate ligament reconstruction in skeletally immature athletes: return to sport, incidence of second surgery, and 2-year clinical outcomes. Am J Sports Med. 2017:45:856-63. 